\title{
\begin{tabular}{l|l} 
Mitraries & DSpace@MIT
\end{tabular}
}

\author{
MIT Open Access Articles
}

\section{Vortex Dynamics in Flow Instabilities and Biomimetic Flows (Prof. Jin S. Chung Award Lecture, 2015)}

The MIT Faculty has made this article openly available. Please share how this access benefits you. Your story matters.

Citation: Triantafyllou, Michael. “Vortex Dynamics in Flow Instabilities and Biomimetic Flows (Prof. Jin S. Chung Award Lecture, 2015)." International Journal of Offshore and Polar Engineering 26, 1 (March 2016): 1-5 (c) 2016 The International Society of Offshore and Polar Engineers

As Published: http://dx.doi.org/10.17736/ijope.2016.jc663

Publisher: International Society of Offshore and Polar Engineers

Persistent URL: http://hdl.handle.net/1721.1/110726

Version: Author's final manuscript: final author's manuscript post peer review, without publisher's formatting or copy editing

Terms of use: Creative Commons Attribution-Noncommercial-Share Alike 


\title{
Vortex Dynamics in Flow Instabilities and Biomimetic Flows Michael S. Triantafyllou \\ Center for Ocean Engineering, Department of Mechanical Engineering \\ Massachusetts Institute of Technology \\ Cambridge, Massachusetts, USA
}

\begin{abstract}
KEY WORDS: Vortex formation, flow instability, flow control, biomimetics.

\section{INTRODUCTION}

Flow-structure interaction is a problem of great importance to ocean and offshore engineering, coastal engineering, and mechanical engineering. Flow mechanisms have been explored with the purpose of reducing loads on large and small structures, cables, risers and hawsers. Theoretical and experimental results derived from studies around structures have found application to a totally new field of study, the biomimetic fluid mechanics, i.e. the mechanisms employed by swimming and flying animals for propulsion and maneuvering. Intriguing similarities in the flow patterns behind swimming animals, for example, and the wake of structures placed in cross-flow, have led to the identification of similar flow mechanisms that can be used to understand the principal properties of both classes of systems. In return, the discoveries made in the study of the swimming of aquatic animals and in bird flight, can be used now in engineering to better control the flow and to devise novel technologies, such as novel sensors and actuators. The proliferation of articles in engineering journals on biomimetics, and the plethora of new biomimetic journals promise that this exchange will continue in the years to come.
\end{abstract}

ABSTRACT

Flow patterns around bodies undergoing flow-induced vibrations and biomimetic flows have intriguing similarities, and similar flow mechanisms can be used to understand their principal properties and find ways to alter their behavior. The long history and extensive study of flow-structure interaction allowed the rapid development of the new field of biomimetic fluid mechanics. In turn, properties and discoveries that were made in the study of the swimming of fish and cetaceans, and bird flight, are entering now the engineering domain, suggesting new ways to control the flow and how to devise new technology, invigorating our profession.

\section{EXPLORING FLOW INSTABILITY MECHANISMS}

One of the most extensively studied topics in flow-structure interaction is the spontaneous formation of the Karman street behind bluff bodies in cross-flow that results in vortex induced oscillations of flexible or flexibly mounted structures (Bearman 1984, Williamson 1996, 2004, Zdravkovish 1997, Triantafyllou et al 2016b). For fixed bodies, and despite the fact that the oncoming cross-flow is steady, the wake flow becomes unstable, generating streets of vortices of alternating-sign vorticity that induce oscillatory loads at the Strouhal frequency. The instability was attributed to the doubly inflected timeaveraged velocity profile, which causes an absolute instability (Bers 1983, Triantafyllou et al 1986, Huerre and Monkewitz 1990), i.e., a flow instability with a preferred frequency that propagates both upstream and downstream, overwhelming the entire flow. The frequency of maximum growth dominates the response and its value is related to the average velocity profile; this is the Strouhal frequency that is related to the width $h$ of the average velocity profile; $f=S t U / h$, where St is the Strouhal number, with a nearly universal value of around 0.16 when related to the width of the wake rather than the diameter of the cylinder. This flow instability constitutes the mechanism through which kinetic energy from the oncoming flow is extracted and then transferred to the body in the form of oscillations.

In the case of a harmonically oscillating foil, mimicking the action of the caudal fin of fish, for example, the role is reversed. Energy supplied by the oscillating foil generates arrays of vortices similar to the Karman street, but of reverse rotating direction than in the case of a bluff body, hence actuating a jet flow rather than a drag wake. Using the same tools of instability analysis, it can be found that a jet flow is accompanied by a convective instability, i.e., an instability that responds at the frequency of excitation with a wavenumber governed by the dispersion relation of the waves as provided by the average velocity profile. At a specific frequency $f$, the spatial growth rate of the waves is maximized; this is the frequency at which the useful energy, in the form of developing thrust, is maximized, and can be used to define a new Strouhal number, also based on the width of the average velocity profile $\mathrm{h}$, which also takes values in a relatively narrow range around $\mathrm{St}=\mathrm{fh} / \mathrm{U}=0.3$. When the excursion of the foil $\mathrm{A}$ 
is used, rather than the width of the velocity profile $h$, a more practically useful approximate Strouhal number is obtained, $\mathrm{St}=\mathrm{fA} / \mathrm{U}$ with values in the range of 0.2 to 0.4 (Triantafyllou et al 1991, 1993, 2000, Anderson et al 1998).

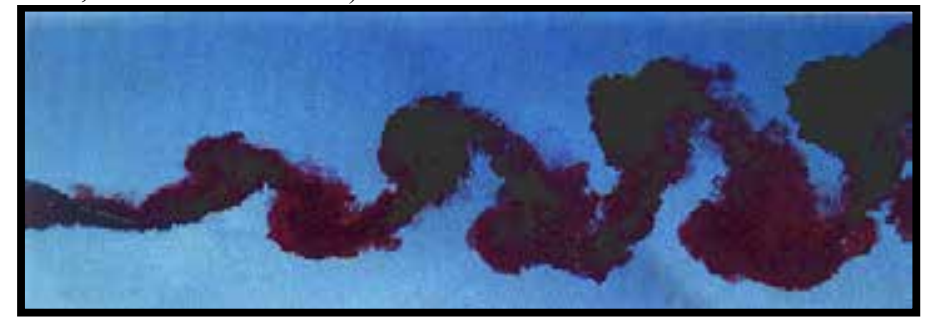

Fig. 1: Jet flow and reverse Karman street visualized through dye behind a tuna-shaped swimming robot (Triantafyllou \& Triantafyllou, 1996).

Further work has shown that birds also power their flights at optimal Strouhal number (Taylor et al 2003), while the notion of flow resonance was established in a variety of flows (Moored et al 2012).

The striking similarity of the flow behind an oscillating foil at optimal frequency of oscillation (reverse Karman street) with the flow behind a bluff body underlines the close similarity of the two phenomena. As already stated, the development of a Strouhal number for fish propulsion was based in the developments for flows behind bluff bodies.

\section{FLOW CONTROL THROUGH VORTEX MANIPULATION AND VORTICITY ANNIHILATION}

The notion of flow resonance helps explain the formation of optimally arranged flow patterns for swimming fish. The principles of vorticity control were investigated by Gopalkrishnan et al (1994) in the case of a foil operating in the wake of a cylinder, to establish ways through which energy contained in the unsteady eddies of a flow can be extracted.

(a)

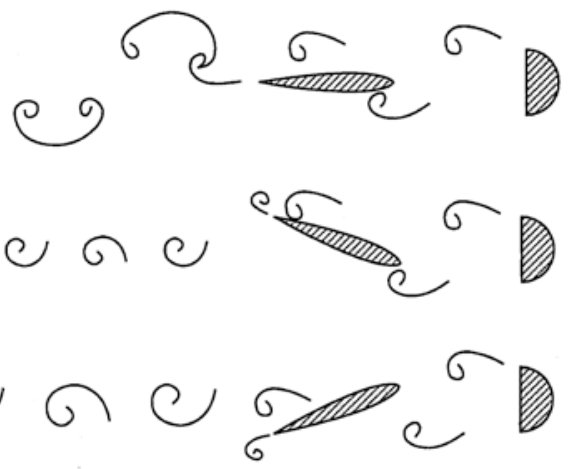

Fig. 2: Principal modes of vorticity control in a model problem consisting of an upstream stationary D-shaped cylinder generating a Karman street, and a downstream foil oscillating at the frequency of the Karman street (Gopalkrishnan et al 2004).

As depicted in Figure 2, there are three principal modes: (a) vortex pairing, whereby in each half-cycle a vortex from the cylinder and a vortex from the foil form a pair, resulting in a laterally expanding wake; (b) vortex annihilation, whereby a cylinder and a foil vortex of opposite vorticity interact resulting in a much weaker wake; and (c) vortex enhancement, whereby a cylinder and a foil vortex of the same vorticity sign provide very strong vortex arrays.
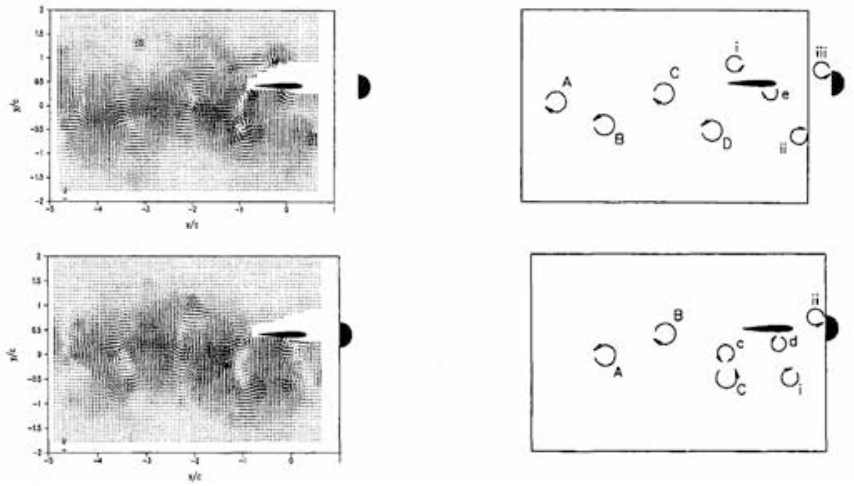

Fig. 3: Vorticity control modes obtained through PIV in a cylinder foil arrangement (Anderson 1996).

Figure 3 illustrates vorticity annihilation (upper figure) and vorticity enhancement (lower figure) as visualized by Anderson (1996) for a Dshaped cylinder and flapping foil in cross flow. As shown thtough simulation earlier (Streitlien et a 1996), vorticity annihilation results in optimal thrust enhancement in a flapping foil, while vorticity enhancement results in higher expended power.
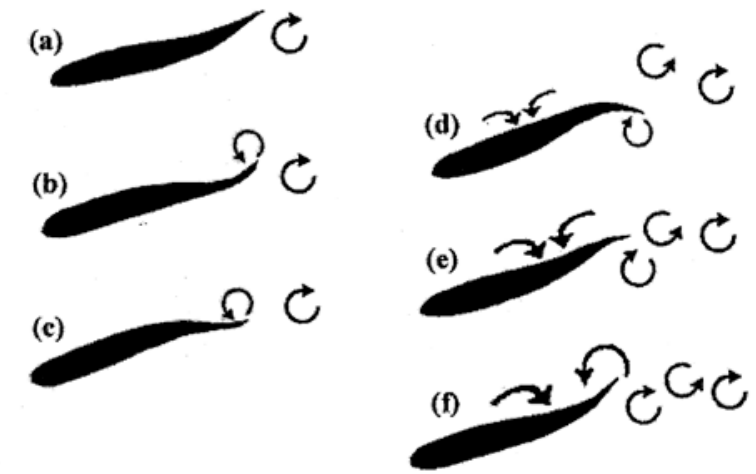

Fig. 4: Vorticity control as employed by swimming fish to minimize expended power (Wolfgang et al 2001).

The principles of vorticity control have been found to apply in a variety of problems, including the swimming and maneuvering of fish. Figure 4 depicts the principal flow patterns around a self-propelled fish. As shown in subfigures (a) through (f), the flow around the body visualized at mid-height cuts, consists of mainly potential flow patterns, resembling those of a traveling water wave. When the unsteady patterns reach the tail they shed to form strong vortices, which are manipulated by the tail to form an optimal reverse Karman street, such as depicted in Figure 1. Vorticity shed by fins is manipulated following the rules of vorticity control in order to gain back some of the expended energy.

\section{THE WHISKERS OF SEALS: LESSONS IN VORTEX INDUCED VIBRATION ELIMINATION}

The whiskers (vibrissae) of the harbor seal are used to detect unsteadiness in the flow so as to locate prey (Denhardt et al 2001; Hanke et al 2010). They have a highly unusual shape as shown in Figure 5, in the form of an elliptical cross-section whose half axes vary sinusoidally along the span of the whisker, resulting in a striking undulatory geometric configuration with average wavelength about 4 
to 6 times the average transverse direction.

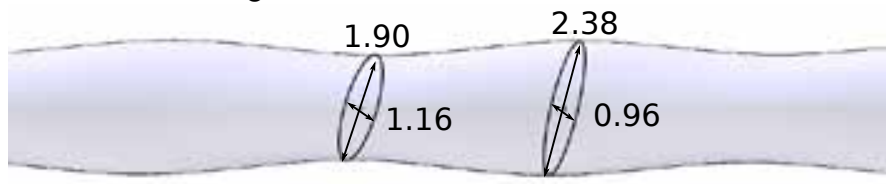

4.00

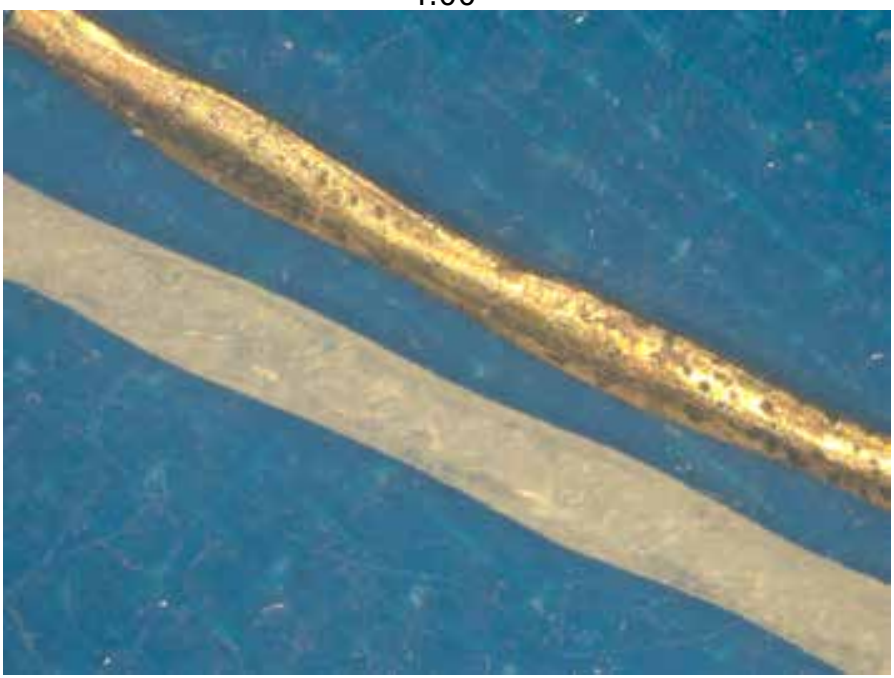

Fig. 5: The striking undulatory shape of the whiskers of a harbor seal. The cross-section is shaped as an ellipse with sinusoidally varying half axes, along the span (upper). Photo of a seal whisker and an artificial whisker model (courtesy of Dr. Ajay Kottapalli).

When the whiskers are placed in cross-flow with the longer axis of their elliptical cross-section along the flow, it is observed that the coherence of vortex shedding is destroyed by the undulatory shape. As shown in Figure 6, a flexible beam with constant elliptical shape exhibits cross-flow VIV response with maximum amplitude to diameter ratio about 0.15 , while the optimally shaped vibrissa exhibits response with ratio about 0.03 (whisker \# 2), and a suboptimal whisker (\# 1) is comparable to that of an ellipse. It is remarkable that the optimal wavelength of the seal whiskers is close to the optimal wavelength found in cylinders of variable diameter to minimize VIV (Lam \& Lin 2009).

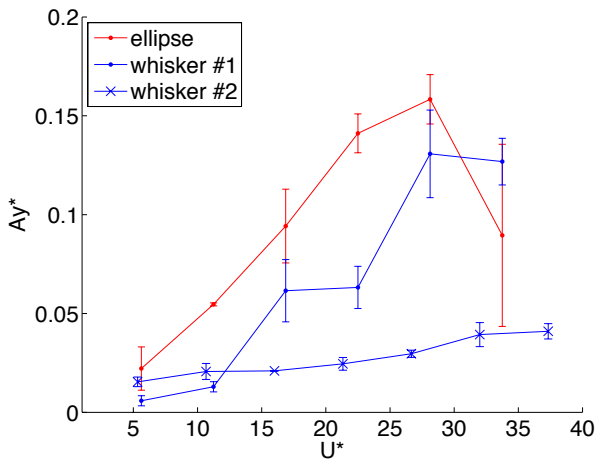

Fig. 6: Vortex-induced vibrations of two differently shaped whiskers of a harbor seal compared with those of an ellipse.

\section{FLOW PATTERNS IN FISH SCHOOLING AND BEHIND MULTI- ELEMENT STRUCTURES}

Fish form large schools, often with hundreds of thousands of individual fish swimming in a sustainable pattern. Each fish produces a vortical wake that interacts with the flow and vortices of other fish. Figure 7 shows the simple case of two swimming fish in tandem, with the downstream fish exploiting the patterns of the upstream fish to reduce its required propulsive energy, often significantly (Martens 2014).

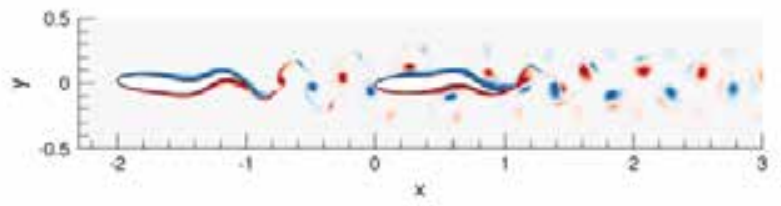

Fig. 7: Snapshot of the vorticity field for two actively swimming fish of length $\mathrm{l}=0.3 \mathrm{~m}$ in tandem at frequency $\mathrm{f}=2.1 \mathrm{~Hz}$, separated by a distance of one body length (Maertens 2014).

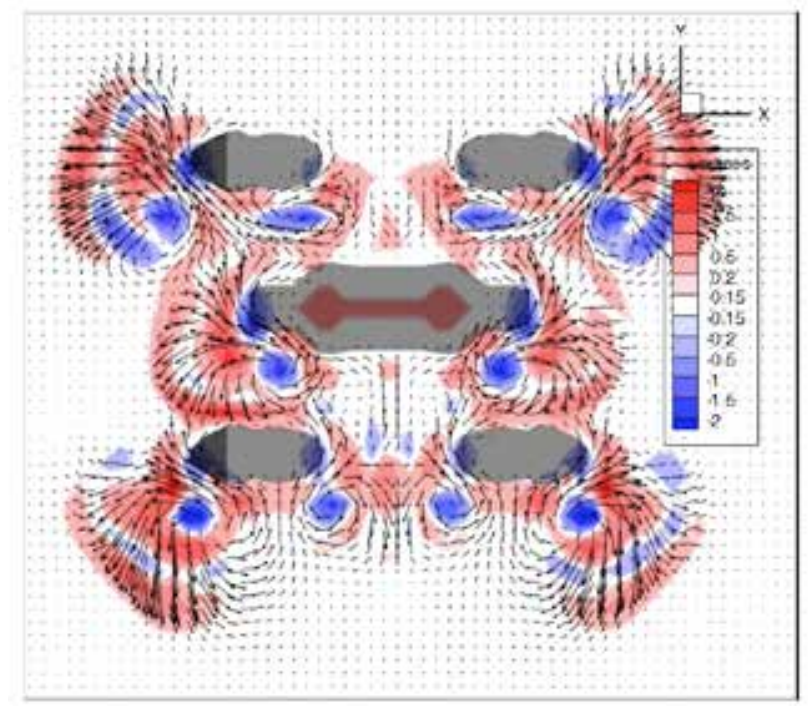

Fig. 8: Velocity and vorticity field at a cross-section of the flow around an ocean structure consisting of five structural elements (depicted in gray). Motion along the y-axis by an amplitude equal to $20 \%$ of the largest width of the structure (Simulation by A. Le Goallec and H. Hans, 2014).

Fish exploit the mechanics of vorticity control (Gopalkrishnan et al 1994) to develop forces on their bodies that are conducive to optimal propulsion; the vortices pair up to either reduce or strengthen their circulation, depending on the desired effect. Such vorticity exploitation has been noted in the studies of Liao et al (2003) on energy extraction by fish behind vortex-shedding cylinders and Beal et al (2006) on energy-extracting foils behind bluff bodies. For schools consisting of a very large number of fish, the wake is altered substantially, to the point of being unrecognizable, i.e. it bears no resemblance to the sum of the individual wakes of fish.

The flow around complex structures in cross flow, consisting of multiple elements, bears close similarities. Although there is no active flow manipulation in this case, unlike the swimming fish, the pairing of vortices by adjacent members can lead to the development of strong jet-like structures, accompanied by a change in vorticity distribution. Figure 8 shows the simulation of a structure consisting of five closely spaced elements, subject to sinusoidal motion of amplitude equal to $20 \%$ of its cross-flow dimension. Vorticity is colored as red (counterclockwise) or blue (clockwise); it is clear that each structural element sheds vortices that pair up with vortices from adjacent members to 
generate jets, which often move at an angle to the direction of motion, which in the figure shown is along the y-direction. The result is that forces develop in both the $\mathrm{x}$ and $\mathrm{y}$ direction, which for the case shown are symmetric about the $\mathrm{y}$ axis (so the total force in the $\mathrm{x}$-direction is zero), but other times it can become asymmetric. More importantly, the vortical patterns draw substantial energy from the oscillations of the structure, resulting in drag coefficients that can be an order of magnitude higher than for a single bluff body of the same projected area, as well as a significantly enhanced added mass coefficient.

\section{SENSING IN FISH PROVIDES A NEW PARADIGM OF SENSORS FOR FLOW CONTROL}

As shown in Figure 6, the seal whiskers exhibit very low VIV when placed in uniform flow. When they are placed in a non-uniform flow, however, then their response changes dramatically. The reason is that their VIV-canceling mechanisms are highly directional, and when placed at an angle of attack with respect to oncoming flow, their VIV increases by an order of magnitude, often exceeding in amplitude their cross-dimension, i.e. comparable to that of a circular cylinder. This property was exploited by Valdivia et al (2012) to develop new sensors capable of detecting even minute wake flows. The physical mechanism driving the whisker response when placed in the wake of an upstream cylinder, as outlined in Beem and Triantafyllou (2015), is a vorticity control mode described as 'slaloming', whereby the whisker oscillates in such a manner as to approach an oncoming cylinder vortex, but, as it is about to touch it, the whisker moves to the opposite direction to encounter the opposite vortex, and so on. This is a flow energy extraction mechanism that was also found in Liao et al (2003) for trout extracting energy for swimming, and in Beal et al (2006) for energy extracting foil, which explain how even dead fish can swim upstream.

Fish utilize their lateral-line system to locate predator or prey and underwater objects (Chagnaud and Coombs 2014). The sensory units are the neuromasts, spread across large portions of the surface of the fish body and consisting of haircells that are embedded into a soft gelatinous material, the cupula. Two types of neuromast sensors exist, superficial neuromast sensors that are freestanding on the surface of the skin exposed to the flow, and canal neuromasts that are embedded inside lateral-line canals along the side of their body.

MEMS (Micro-electro-mechanical systems) technology offers unique advantages for developing inexpensive, low-power arrays of microflow sensors emulating the function of the lateral line (Triantafyllou et al 2016b).
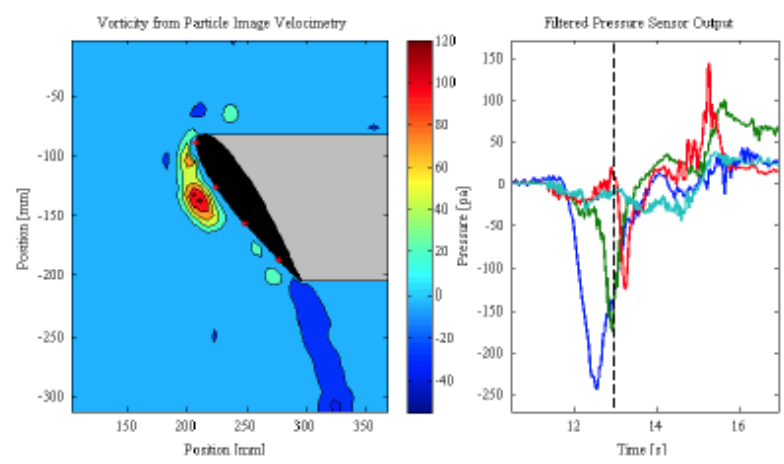

Fig. 9: Vortex detection on a stalling foil at an angle of attack of 35 deg and moving at $0.3 \mathrm{~m} / \mathrm{s}$, using four pressure sensors (red dots). Vertical dotted line at right corresponds to frame at left (Fernandez et al 2011, Dusek et al 2013).
Two different types of sensors are fabricated - the superficial haircell sensors which perform flow velocity sensing and the canal encapsulated haircell sensors which perform flow acceleration sensing. The flow sensor consists of an LCP membrane with gold strain gauges deposited on it and a highaspect ratio Si60 haircell fabricated by stereolithography. Flexible arrays of polymer MEMS haircell sensors are fabricated and encapsulated in polydimethylsiloxane (PDMS) canal structures. The superficial haircell sensors are calibrated in water tunnel for flow velocity sensing. The sensors demonstrated a sensitivity of $0.022 \mathrm{~V} /(\mathrm{ms}-1)$. Methods to further increase the sensitivity have resulted in outstanding results, nearly matching the sensitivity of live fish (Asadnia et al 2016).

The development of inexpensive, low power arrays of velocity and pressure sensors can be a game-changing technology as it will allow new experimentation methods in fluid mechanics, and, more significantly, ways to continuously monitor the hydrodynamic performance of marine vehicles.

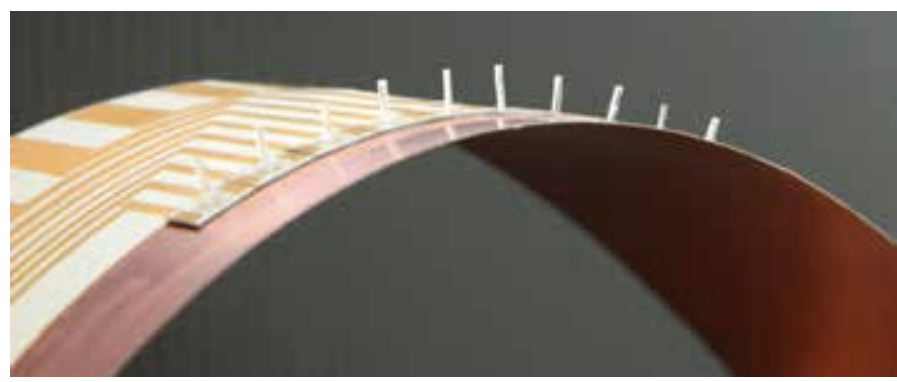

Fig. 10: A flexible velocity sensor array consisting of 10 LCP neuromast-like sensors (Kottapalli et al 2015, Asadnia et al 2016).

\section{CONCLUSIONS}

For over 25 years now biomimetic fluid mechanics have drawn inspiration and used methods from conventional fluid mechanics. In return, they offer today new insight on the generation and control of unsteady flows, which can benefit engineering applications. In addition, inspiration is drawn from live animals on mechanisms of sensing and flow control that provide new insight for engineered systems, as well as to design novel sensors and actuators that constitute a completely new and untapped paradigm of technology development.

\section{ACKNOWLEDGEMENTS}

The paper is written for the 2015 J.S. Chung lecture and award. The author acknowledges with gratitude the award, as he has had the privilege of a long collaboration with Prof. Chung, whose contributions to the field of ocean engineering are invaluable, including the establishment of a vibrant professional society, ISOPE, whose annual conferences attract some of the best publications in the field.

\section{REFERENCES}

Anderson, JM, 1996, "Vorticity control for efficient propulsion", PhD Thesis, Joint MIT-WHOI Program, Cambridge, MA, February 1996.

Anderson, JM, Streitlien, K, Barrett, DS, \& Triantafyllou, MS, 1998, "Oscillating foils of high propulsive efficiency", Journal of Fluid Mechanics, 360, 41-72. 
Asadnia, M, Kottapalli, A, Miao, JM, \& Triantafyllou, MS, 2016, "Artificial fish skin of self-powered MEMS hair cells for sensing hydrodynamic flow phenomena", J. R. Soc. Interface (to appear).

Beal, DN, Hover, FS, Triantafyllou, MS, Liao, JC, \& Lauder, GV, 2006, "Passive propulsion in vortex wakes" Journal of Fluid Mechanics 549, 385-402.

Bearman, PW, 1984, "Vortex shedding from oscillating bluff bodies", Annu. Rev. Fluid Mech. 16, 195-222.

Beem, H, Triantafyllou MS, 2015, "Wake-induced 'slaloming' response explains exquisite sensitivity of seal whisker-like sensors", J. Fluid Mech., 783, 306-322.

Bers, A, 1983, "Basic Plasma Physics I", in Handbook of Plasma Physics (ed. M.N. Rosenbluth \& R.Z. Sagdeev), vol. 1, chapt. 3.2. North Holland.

Chagnaud, BP, Coombs, S, 2014, "Information encoding and processing by the peripheral lateral line system" In Coombs et al. 2014, pp. 151-94.

Coombs, S, Bleckmann, H, Fay, RR, Popper, AN, eds., 2014, "The Lateral Line System", New York: Springer.

Dehnhardt, G, Mauck, B, Hanke, W, \& Bleckmann, H, 2001, "Hydrodynamic trail- following in harbor seals (phoca vitulina)", Science 293 (5527), 102-104.

Dusek, J, Kottapalli, AGP, Woo, ME, Asadnia, M, Miao, JM, Triantafyllou, MS, 2013, "Development and testing of bio-inspired microelectromechanical pressure sensor arrays for increased situational awareness for marine vehicles", Smart. Mater. Struct. 22, $014002-014015$.

Fernandez VI, Maertens A, Yaul FM, Dahl J, Lang JH, Triantafyllou MS, 2011, "Lateral-line inspired sensor arrays for navigation and object identification", Marine Technology Society Journal, 45:130146.

Gopalkrishnan, R, Triantafyllou, MS, Triantafyllou, GS, \& Barrett, DS, 1994, "Active Vorticity Control in a Shear Flow Using a Flapping Foil", Journal of Fluid Mechanics, 274, 1-21.

Hanke, F, Leder, A, and Dehnhardt, G, 2010 "Harbour seal vibrissa morphology suppresses vortex induced vibrations", J. Exp. Bio., 213, pp. 2665-2672.

Huerre P, and Monkewitz P, 1990, "Local and global instabilities in spatially developing flows", Ann. Rev. Fluid Mech., 22, 473-537.

Kottapalli, AGP, Asadnia, M, Haghighi, R, Cloitre, A, Valdivia y Alvarado, P, Miao, JM, \& Triantafyllou, MS, 2015, "MEMS sensors for assessing flow-related control of an underwater biomimetic robotic stingray", Bioinspiration \& Biomimetics, 10 (3).

Lam, K, \& Lin, YF, 2009, "Effects of wavelength and amplitude of a wavy cylinder in cross-flow at low Reynolds numbers", J. Fluid Mech., 620, 195-220.

Liao, JC, Beal, DN, Lauder, GV, \& Triantafyllou, MS, 2003, "Fish exploiting vortices use less muscle", Science, 302 (5650), 15661569, November 28, 2003.

Maertens A, 2014, "Fish swimming optimization and exploiting multibody hydrodynamic interactions for underwater navigation", $\mathrm{PhD}$ Thesis, MIT, Cambridge, Mass.

Moored, KW, Dewey, PA, Smits, AJ, \& Haj-Hariri H, 2012, "Hydrodynamic resonance as an underlying principle of efficient unsteady propulsion", J. Fluid Mech., 708, 329-348.

Streitlien, K, Triantafyllou, GS, \& Triantafyllou, MS, 1996, "Efficient foil propulsion through vortex control", AIAA Journal, 34 (11), 2315-2319.

Taylor, GK, Nudds, RL, and Thomas, ALR, 2003, "Flying and swimming animals cruise at a strouhal number tuned for high power efficiency", Nature (London), 425 (6959), 707-711.

Triantafyllou, GS, Triantafyllou, MS, \& C. Chryssostomidis, C, 1986, "On the formation of vortex streets behind stationary cylinders",
Journal of Fluid Mechanics, 170, 461-477.

Triantafyllou, MS, Triantafyllou, GS, \& Gopalkrishnan, R, 1991, "Wake Mechanics for Thrust Generation in Oscillating Foils", Physics of Fluids A, 3 (12), 2835-2837.

Triantafyllou, GS, Triantafyllou, MS, \& Grosenbaugh, MA, 1993, "Optimal Thrust Development in Oscillating Foils with Application to Fish Propulsion", Journal of Fluids and Structures, 7, 205-224.

Triantafyllou, MS, \& Triantafyllou, GS, 1995, "An Efficient Swimming Machine", Scientific American, 272 (3), 64-70.

Triantafyllou, MS, Weymouth, G, and Miao, J, 2016a, "Biomimetic survival hydrodynamics and flow sensing", Annual Reviews of Fluid Mechanics, 48 (in the press).

Triantafyllou, MS, Bourguet R, Dahl, J, Modarres-Sadeghi. Y, 2016b, "Vortex-Induced Vibrations", in Handbook of Ocean Engineering, ed. Ertekin C., (in the presss).

Valdivia y Alvarado, P, Subramaniam, V, and Triantafyllou, MS, 2012 "Design of a Bio-Inspired Whisker Sensor for Underwater Applications", Proc. IEEE Sensors, Taipei, Taiwan.

Williamson, CHK, 1996, "Vortex shedding in the cylinder wake", Annual Rev. Fluid Mech., 28 , 477 - 539.

Williamson, CHK, \& Govardhan, R, 2004, "Vortex-induced vibrations", Annual Review of Fluid Mechanics 36 (1), 413-455.

Zdravkovich, MM, 1997, "Flow around circular cylinders, vol. 1: fundamentals", Oxford, UK: Oxford University Press. 